\title{
Disease Note
}

\section{Diseases Caused by Fungi and Fungus-Like Organisms}

First Report of Anthracnose on Jerusalem Cherry Caused by Colletotrichum liaoningense in Shandong, China

Yun Liu, ${ }^{1, \dagger}$ Fei An, ${ }^{2}$ Yujiao Zhang, ${ }^{1}$ Cuicui Fu, ${ }^{1}$ Yuebo $\mathrm{Su}^{1}$

${ }^{1}$ Jinan City Garden and Forestry Greening Bureau, Jinan Forest Farm, Jinan, Shandong, 250013, China

${ }^{2}$ Qilu Institute of Technology, Jinan, Shandong, 250200, China

Plant Dis. 105:2248, 2021; published online as https://doi.org/10.1094/PDIS01-21-0124-PDN. Accepted for publication 28 February 2021.

Jerusalem cherry (Solanum pseudocapsicum), which belongs to the genus Solanum and the family Solanaceae, possesses high ornamental value and is widely cultivated as an indoor ornamental due to its bright red berries at maturity (Xu et al. 2018). In September 2019, leaf spot was detected on Jerusalem cherry plants in Yuxiu Park, Shizhong district, Jinan, Shandong Province. Field surveys were done in a 1/15-ha park. Disease incidence was estimated at approximately $18 \%$ across the survey area. Foliar symptoms began as small white spots. As the disease progressed, lesions expanded and merged, and developed into large irregular white spots, with a pale gray edge. Finally, lesions were densely distributed throughout the leaves. To isolate the pathogen, 20 leaf tissues $(5 \times 5 \mathrm{~mm})$ were cut from the border between diseased and healthy tissue, surface disinfected in $75 \%$ alcohol for $15 \mathrm{~s}$, soaked in $0.1 \%$ mercuric chloride for $1 \mathrm{~min}$, washed with sterile distilled water three times, and cultured on potato dextrose agar (PDA) at $25^{\circ} \mathrm{C}$. Nineteen fungal isolates were obtained and were single spored to obtain pure cultures. The colony of LCL7, a representative isolate, on PDA was initially white to orange but turned black after 3 to 4 days of incubation with black conidial masses. Conidia were single-celled, hyaline, straight, cylindrical, apex obtuse, and ranged from 13.4 to $18.3 \times 3.2$ to $4.9 \mu \mathrm{m}(n=50)$. To validate the species identification, the rDNA internal transcribed spacer (ITS) region (White et al. 1990) and the partial sequences of glyceraldehyde3 -phosphate dehydrogenase (GAPDH), actin (ACT), $\beta$-tubulin (TUB2), and chitin synthase (CHS-1) (Damm et al. 2019; He et al. 2019) were amplified and sequenced. The ITS, GAPDH, ACT, TUB2, and CHS- 1 sequences of isolate LCL7 were submitted to GenBank (MW221320, MW227217,
MW227218, MW227219, and MW266988, respectively). ITS, ACT, TUB2, and CHS-1 BLAST showed 99 to $100 \%$ homology with sequences of Colletotrichum liaoningense (ITS, $100 \%$ to MH636504; ACT, $100 \%$ to MH622582; TUB2, 99.56\% to MH622714; CHS-1, 99.33\% to MH622446; respectively), although GAPDH showed $93.98 \%$ homology with sequence MH681383 (234/249 bp). A neighbor-joining tree based on concatenated sequences of the five genes was constructed using MEGA7.0. The results showed the isolate was closely related to $C$. liaoningense. Based on morphological and molecular characteristics, the isolate LCL7 was identified as C. liaoningense. Pathogenicity tests were performed by spraying a conidial suspension $\left(1 \times 10^{5}\right.$ conidia/ml $)$ on ten 2 -year-old healthy Jerusalem cherry plants. Ten other plants with sterile water served as controls. All samples were incubated in a growth chamber at $25 \pm 2^{\circ} \mathrm{C}$ and transparent plastic bags to keep relative humidity high for 2 days. All inoculated plants showed symptoms similar to those observed in the field after 21 days, but no disease occurred on control plants. The same fungus was successfully reisolated from inoculated leaves and reidentified based on morphology and molecular characteristics, and the fungus was not isolated from the control plants, thus confirming Koch's postulates. Pathogenicity tests were repeated twice. C. liaoningense can cause anthracnose in chili and mango in China (Diao et al. 2017; Li et al. 2019). To our knowledge, this is the first report of anthracnose on Jerusalem cherry caused by $C$. liaoningense in China, which influences ornamental value and reduces market value. Identification of the causes of the disease will help develop effective strategies for managing this disease.

References:

Damm, U., et al. 2019. Stud. Mycol. 92:1.

Diao, Y., et al. 2017. Persoonia 38:20.

He, L., et al. 2019. Plant Dis. 103:34.

Li, Q., et al. 2019. Sci. Rep. 9:18891.

White, T. J., et al. 1990. Page 315 in: PCR Protocols: A Guide to Methods and Applications. Academic Press, San Diego, CA.

Xu, H., et al. 2018. PeerJ 6:e4424.

The author(s) declare no conflict of interest.

e-Xtra

Keywords: Jerusalem cherry, anthracnose, Colletotrichum liaoningense

${ }^{\dagger}$ Indicates the corresponding author.

Y. Liu; liuyun54335@163.com 